\title{
Variations in the anatomical dimensions of the mandibular ramus and the presence of third molars: its effect on the sagittal split ramus osteotomy
}

\author{
J. Beukes ${ }^{1}$, J. P. Reyneke $e^{1,2,3,4}$, P. J. Becker ${ }^{5,6}$
}

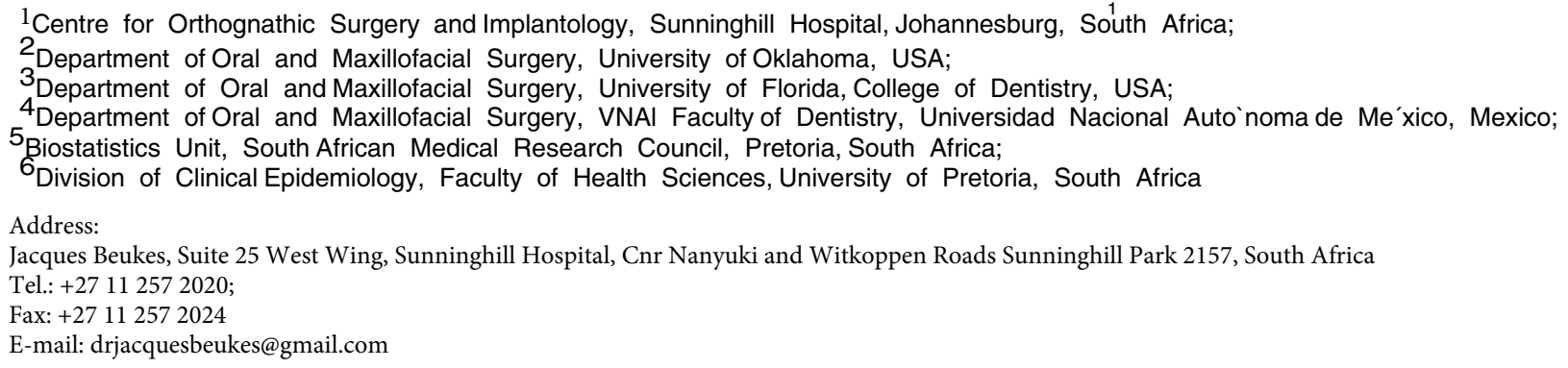

Key words: bilateral sagittal split osteotomy (BSSO); unfavourable splits; mandibular anatomy; third molars.

\begin{abstract}
The authors undertook a prospective study to evaluate the influence that the anatomical dimensions of the ramus of the mandible and the presence of lower third molar teeth may have on the sagittal split ramus osteotomy. The anatomical dimensions measured included the width of the anterior mandibular ramus, the height of the corpus posterior to the second mandibular molar and the antero-posterior anatomical position of the lingual. The influence that these dimensions of the mandible may have on the successful splitting of the mandibular ramus wasThe effect that the presence of wisdom teeth had on the difficulty of the procedure was also investigated. This study found that, unlike the presence of third molars, there was no single anatomical measurement that contributed to the level of difficulty of the sagittal split osteotomy. For descriptive purposes the authors proposes a classification of the four typical patterns of unfavourable splits.
\end{abstract}

Since a report in 1907 by VP Blair ${ }^{1}$ on surgical repositioning of the mandible for the correction of a prognathic mandible, the technique for the surgical correction of dentofacial deformities has developed into a well defined science and a fascinating art form.

In 1957 Trauner and Obwegeser ${ }^{2}$ described a surgical procedure which involved repositioning the mandible by splitting the mandibular ramus in a sagittal plane. This technique enabled the surgeon to establish occlusal function and improved facial aesthetics by repositioning the mandible either anteriorly or posteriorly. The technique was later modified by Dal Pont ${ }^{3}$ and further refined by Hunsuck in $1968^{4}$ and Epker in 1977.5

Bilateral sagittal ramus osteotomy is currently the most popular surgical procedure for the correction of dentofacial deformities involving the mandible. ${ }^{6}$ The ingenuity of the procedure, development of specialized instruments and the improvement in surgeons' experience and skills has made it possible to achieve surgical goals quickly and atraumatically with predictable results. ${ }^{7,8}$

As with any surgical procedure, intraand postoperative complications may 
occur. Complications associated with the surgical technique when performing the sagittal split osteotomy are well documented in the literature, some of the most common being unfavourable fractures of either the distal or proximal segment, damage to the inferior alveolar nerve, failure of fixation and condylar sag. ${ }^{6-12}$

Although the anatomy of the posterior part of the mandible lends itself to the surgical design, it has become evident that the specific anatomy of each individual mandibular ramus differs which may play a significant role in the ease of splitting the ramus. Various technical factors could influence the successful completion of the split and there are several important anatomical structures that should be considered when the split is performed. ${ }^{12}$ Maintaining the mandibular condyle/fossa relationship forms the basis for the achievement of the new planned occlusion. Correct positioning of the condyles in the fossae during surgery is therefore mandatory and is certainly challenging. The involvement of the inferior alveolar nerve should be carefully considered and the neurovascular bundle must be preserved during surgery to prevent postoperative neurosensory deficit of the lower lip and chin.

The anatomical shape of the mandible, bone thickness, height of the ramus, the position of the lingula and presence of an impacted third molar tooth are all important factors which influence the successful execution of the sagittal ramus split. Experience has shown that there are four typical patterns of unfavourable fractures that may occur during the surgery. For descriptive purposes the authors propose a classification of unfavourable splits during the sagittal split osteotomy.

Type 1 is buccal plate fracture. Fracture of the distal aspect of the proximal segment of the mandibular ramus or buccal plate usually occurs at the initial stage of the split. This complication may involve only part of the buccal plate (Fig. 1a). Type 2 is buccal plate fracture including the coronoid process. Fracture of the proximal segment may extend further superiorly including the coronoid process of the mandible (Fig. 1b). Types 1 and 2 fractures usually occurs following failure to include the lingual cortex in the vertical part of the osteotomy (Fig. 2). A wide and rounded mandibular lower border makes inclusion of the lingual cortex in the vertical osteotomy difficult.

Type 3 is retromolar fracture. The retromolar area of the distal segment of the mandible is often thin and fragile. The fragility of the bone may be further

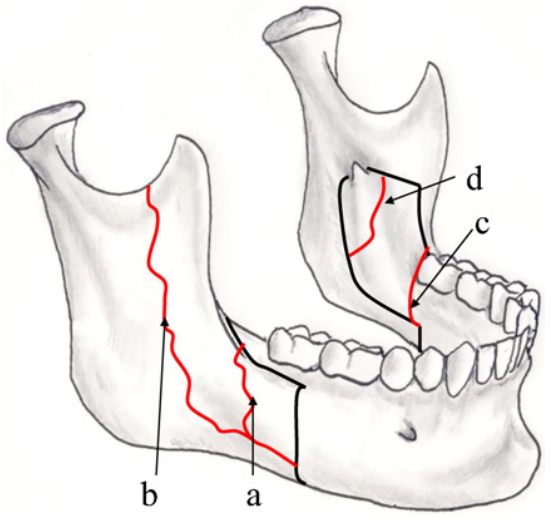

Fig. 1. Patterns of unfavourable splits: (a) buccal plate fracture on the distal aspect of the proximal segment; (b) buccal plate fracture extending to the coronoid notch; (c) fracture of the proximal aspect of the distal fragment; (d) short fracture of the lingula.

increased by the presence of an impacted third molar tooth in the line of the osteotomy (Fig. 1c). The integrity of the bone in this segment plays an important role when fixating the segments and is essential for placement of bicortical screw rigid fixation. Fracture of this segment will limit placement of bicortical screws and increase the risk of fixation failure. An alternative fixation method such as the use of plate fixation should be considered in this case. A rare complication of bilateral postoperative fracture of the lingual plates in the retromolar area leading to severe bilateral open bite malocclusion has been reported. ${ }^{11}$

Type 4 is short fracture of the lingula. The position of the lingula, the mandibular foramen and the depth of the fossa posterior to the lingula are additional anatomical structures which demand consideration when planning and performing the sagittal

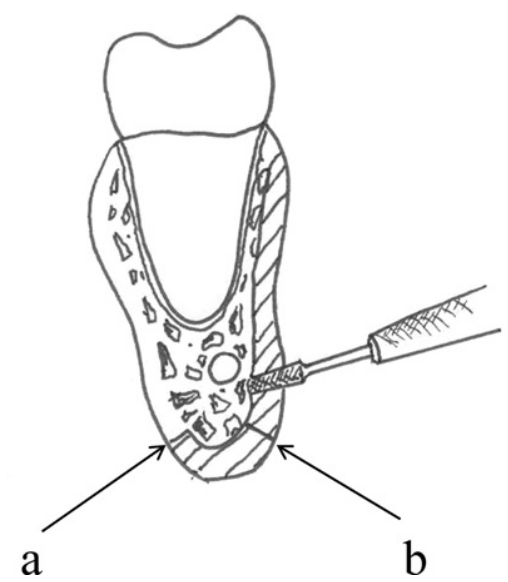

Fig. 2. Vertical osteotomy in the mandibular corpus. (a) The osteotomy should be extended medially to include the lingual cortex of the mandible. (b) If the osteotomy does not include the lingual cortex it will lead to a fracture of the buccal cortex. split ramus osteotomy. The modification to the sagittal split osteotomy by Hunsuck ${ }^{4}$ and Epker ${ }^{5}$ allows the surgeon to split the mandible anterior to the posterior border of the mandible. The osteotomy, according to this modification, runs from just posterior to the lingula, downwards on the lingual side of the ramus towards the lingual aspect of the vertical osteotomy (Fig. 1d).

Failure to extend the horizontal osteotomy to just posterior to the lingula into the fossa, on the medial side of the ramus, will lead the osteotomy to fracture anterior to the lingula. The lingula, foramen and superior part of the inferior alveolar canal, will now remain attached to the proximal segment (Fig. 1d). If this complication does occur, the attached bony canal, containing the neurovascular bundle, should be carefully dissected from the proximal segment. This may further jeopardize the neurosensory prognosis. Repositioning of the distal segment without releasing the inferior alveolar neurovascular bundle from the bone will stretch the nerve adding to the risk of neurosensory deficit of the lower lip and chin on the affected side.

\section{Patients and methods}

74 patients volunteered to take part in the study (permission from the Human Medical Research Ethics Committee was obtained: M0050341). Data were captured over a period of 8 months from consecutive patients who had received sagittal split osteotomies. The same surgeon performed 147 sagittal split osteotomies on 74 patients. 73 patients received bilateral osteotomies while a unilateral osteotomy was performed on one patient (73 osteotomies were performed on the right side and 74 osteotomies on the left side). 
$59 \%$ of patients included in the study were female (44) and $42 \%$ were males (30). The age of the patients ranged from 13 to 53 years with an average age of 26 years at the time of surgery.

Each patient's surgical treatment was comprehensively planned and included cephalometric surgical objectives and model surgery. A standard surgical routine allowed the surgical team to anticipate each step, prevent intra-operative uncertainty and helped to eliminate postoperative complications.

During surgery, a single experienced operator measured the width of the mandibular rami at the level of the horizontal osteotomy (the height of the lingula) while the width of the mandibular bodies were measured just posterior to the second molar tooth (Fig. 3). Measurements were performed using a calliper.

Additional measurements were performed on a standard panoramic radiograph taken on the same X-ray machine (Planmeca 2002 cc Prolive) by the same radiographer and included: the distance from the lower border of the mandible to the apex of the distal root of the lower second molar tooth; the height of the corpus of the mandible $3 \mathrm{~mm}$ posterior to the lower second molar; the distance from the anterior border of the ramus to the anterior border of the lingual; and the antero-posterior dimension of the ramus at the level of the lingula (Fig. 4).

The presence of an impacted/unerupted/ erupted lower third molar tooth was noted.

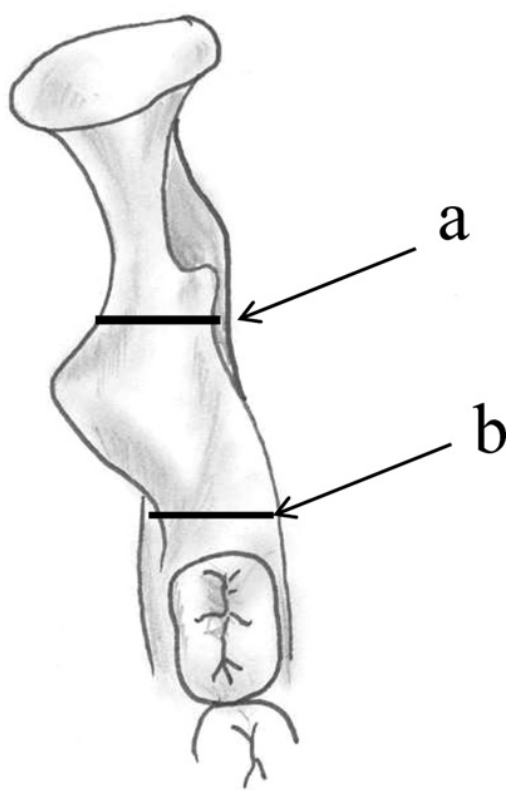

Fig. 3. Intraoperative measurements included (a) the width of the ramus at the level of the lingual and (b) the width of the mandible $3 \mathrm{~mm}$ posterior to the second lower molar.

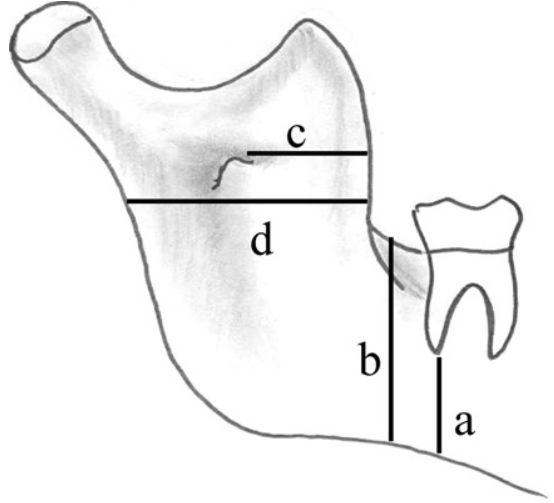

Fig. 4. Measurements on the panoramic radiograph. (a) The distance from the lower border of the mandible to the apex of the distal root of the lower second molar; (b) height of the corpus of the mandible $3 \mathrm{~mm}$ posterior to the lower second molar; (c) distance from the anterior border of the ramus to the anterior border of the lingual; and (d) antero-posterior dimension of the ramus of the mandible.

Third molars prevented from normal eruption by either the second molar tooth or the mandibular ramus were classified as impacted, whereas third molars which appeared to have adequate space for eruption were classified as unerupted.

Following each procedure the surgeon evaluated and graded the difficulty of splitting the mandibular ramus as follows. It was labelled Grade 1 if once the straight osteotome was placed into the osteotomy line at the superior border and the Reyneke splitter placed into the vertical osteotomy at the lower border and rotated the segments split apart without difficulty. It was labelled Grade 2 if there was a tendency to a greenstick and/or bad split and inferior alveolar nerve involvement, requiring dissection of the neurovascular bundle or canal. The split required redefining the osteotomies to ensure correct splitting.

\section{Statistical analysis}

Six mandibular measurements were made on both the left and right side. These measurements were summarized by grade of difficulty using descriptive statistics mean and standard deviation. Group comparisons were done using Students' two couple $t$-test and $p$-values along with 95\% confidence intervals for the mean difference between groups were reported. Groups were also compared with respect to the distribution of wisdom teeth (absent, erupted, unerupted, impacted), using Fisher's exact test. Testing was done at the 0.05 level of significance.

\section{Results}

Grades of difficulty differed significantly $(p=0.015)$ with respect to measurement
5 -L and significantly $(p=0.071)$ with respect to measurement 6-R (Table 1).

Grades of difficulty differed significantly $(p=0.003)$ with respect to wisdom teeth distribution; in particular for the difficult group (Grade 2). This group has a high proportion of unerupted $(33.33 \%)$ and impacted $(21.22 \%)$ wisdom teeth, while the easier group (Grade 1) only had $12.5 \%$ unerupted and $2.5 \%$ impacted wisdom teeth present (Table 2).

The patients undergoing Grade 1 sagittal splits had a mean age of 29 years (1453 years), whereas those in Grade 2 had a mean age of 21 years (13-50 years). This confirms that difficult splits tend to occur in a younger age group. ${ }^{13}$

\section{Discussion}

In the last four decades sagittal split osteotomy has evolved from a life threatening procedure to, in some cases, outpatient surgery.

In this prospective study no unfavourable splits of the mandible were reported. The surgeons' experience and skill may be a factor in this result. Approaching each patient with a standard surgical technique that involves appropriate treatment planning, a consistent surgical routine and the use of modern well designed instrumentation helps to minimize intra-operative and postoperative complications.

Although no intra-operative or postoperative bad splits occurred in this study, there were a number of osteotomies that proved to be technically more difficult than others. The presence of lower third molars influenced the level of difficulty. In most instances where third molars were present during the time of surgery an effort was made to retain these teeth. Removal of 
Table 1. Comparison of difficulty grade 1 and difficulty grade 2 treatment groups with respect to mean mandibular measurements (mm).

\begin{tabular}{|c|c|c|c|c|c|c|c|}
\hline \multirow{3}{*}{ Measurement } & & \multicolumn{4}{|c|}{ Grade of difficulty } & \multirow{3}{*}{$p$-Value ${ }^{*}$} & \multirow{3}{*}{$\begin{array}{l}\text { 95\% C.I. for } \\
\text { difference }\end{array}$} \\
\hline & & \multicolumn{2}{|c|}{ Grade 1} & \multicolumn{2}{|c|}{ Grade 2} & & \\
\hline & & Mean (SD) & $N$ & Mean (SD) & $N$ & & \\
\hline \multirow{2}{*}{$\begin{array}{l}\text { 1. Lower border of the body of the mandible to the } \\
\text { apex of the distal root of the lower second molar. }\end{array}$} & $\mathrm{R}$ & $12.97(3.06)$ & 45 & $13.78(4.17)$ & 28 & 0.34 & $(-2.5 ; 0.88)$ \\
\hline & $\mathrm{L}$ & $13.47(3.22)$ & 48 & $13.07(3.41)$ & 26 & 0.61 & $(-1.19 ; 1.99)$ \\
\hline \multirow{2}{*}{$\begin{array}{l}\text { 2. Height of the body of the mandible } 3 \mathrm{~mm} \text { posterior } \\
\text { to the lower second molar. }\end{array}$} & $\mathrm{R}$ & $28.76(4.10)$ & 45 & $30.46(4.81)$ & 28 & 0.11 & $(-3.80 ; 0.41)$ \\
\hline & $\mathrm{L}$ & $28.57(4.24)$ & 48 & $28.96(4.79)$ & 26 & 0.71 & $(-2.54 ; 1.77)$ \\
\hline \multirow{2}{*}{$\begin{array}{l}\text { 3. Distance from the anterior border of the ramus to } \\
\text { the anterior border of the lingula. }\end{array}$} & $\mathrm{R}$ & $17.08(3.34)$ & 45 & $18.00(3.07)$ & 28 & 0.24 & $(-2.46 ; 0.64)$ \\
\hline & $\mathrm{L}$ & $16.07(2.92)$ & 48 & $16.59(3.09)$ & 26 & 0.47 & $(-1.97 ; 0.92)$ \\
\hline \multirow{2}{*}{$\begin{array}{l}\text { 4. Antero-posterior dimension of the ramus at the } \\
\text { level of the lingula. }\end{array}$} & $\mathrm{R}$ & $35.74(3.90)$ & 45 & $36.03(4.85)$ & 28 & 0.77 & $(-2.35 ; 1.76)$ \\
\hline & $\mathrm{L}$ & $33.83(4.05)$ & 48 & $32.92(4.39)$ & 26 & 0.37 & $(-1.11 ; 2.93)$ \\
\hline \multirow{2}{*}{$\begin{array}{l}\text { 5. Width of the mandible posterior to the lower } \\
\text { second molar. }\end{array}$} & $\mathrm{R}$ & $15.87(2.22)$ & 45 & $16.33(1.91)$ & 28 & 0.36 & $(-1.47 ; 0.55)$ \\
\hline & $\mathrm{L}$ & $15.96(2.29)$ & 48 & $17.28(1.94)$ & 26 & 0.01 & $(-2.37 ; 0.26)$ \\
\hline \multirow{2}{*}{$\begin{array}{l}\text { 6. Width of the mandibular ramus at the level of } \\
\text { the lingula. }\end{array}$} & $\mathrm{R}$ & $7.43(1.61)$ & 45 & $8.19(1.74)$ & 28 & 0.06 & $(-1.56 ; 0.03)$ \\
\hline & $\mathrm{L}$ & $8.01(1.50)$ & 48 & 8.69 (2.32) & 26 & 0.15 & $(-1.57 ; 0.20)$ \\
\hline
\end{tabular}

${ }^{*} p$-Value associated with Student's two-sample $t$-test and $p<0.05$ denotes a statistically significant difference between difficulty groups.

Table 2. Comparison of Grade 1 and Grade 2 treatment groups with respect to the distribution of wisdom teeth.

\begin{tabular}{lccr}
\hline \multirow{2}{*}{ Third molars } & \multicolumn{2}{c}{ Grade of difficulty } & \multirow{2}{*}{ Total } \\
\cline { 2 - 3 } & Grade 1 & Grade 2 & \\
\hline Absent & $29(70.73 \%)$ & $12(36.36 \%)$ & $41(55.41 \%)$ \\
Erupted & $6(14.63 \%)$ & $3(9.09 \%)$ & $9(12.16 \%)$ \\
Unerupted & $5(12.20 \%)$ & $11(33.33 \%)$ & $16(21.62 \%)$ \\
Impacted & $1(2.44 \%)$ & $7(21.21 \%)$ & $8(10.81 \%)$ \\
Total & $41(100 \%)$ & $33(100 \%)$ & $74(100 \%)$ \\
\hline
\end{tabular}

the third molar weakens the retromolar part of the distal segment and also complicates placement of bicortical screw fixation and may even lead to Type 2 unfavourable splits. The authors anticipated that other factors such as the height and the width of the corpus of the mandible and the width and thickness of the ramus would influence the difficulty level but this study showed that no single measurement influenced the difficulty of the sagittal split. Experienced surgeons are often able to recognize anatomical limitations early, adjust their surgical technique appropriately and thus limit the possible complications associated with the above mentioned factors.

Reyneke et al., ${ }^{13}$ Precious et al. ${ }^{9}$ and Mehra et al. ${ }^{14}$ indicated an increased risk of an unfavourable split with unerupted third molar teeth. Previous studies have recommended the removal of unerupted lower third molar teeth at least 6 months prior to the surgical procedure. ${ }^{11}$ The authors think that 9 months is more appropriate. They have found that when performing a sagittal split osteotomy after 6 months the regenerative bone in the tooth socket remains soft. When a patient presents for orthognathic surgery with unerupted or impacted third molars, the surgeon has three options: surgical removal of the third molars, increasing the orthognathic treatment time by $6-9$ months; performing the sagittal split osteotomy and leaving the third molars in situ, which will subject the patient to a second procedure later for removal of the third molars; and removing the third molars at the same time as the sagittal split osteotomy, acknowledging the increased risk of a unfavourable split or even inferior alveolar nerve damage.

In conclusion, even though there were no statistically significant differences between the dimensions of the mandible easy osteotomies, experience has shown that cases with extreme anatomical variations, for example an extremely narrow mandible or small mandibular height does contribute to the difficulty of the sagittal split osteotomy and requires a more cautious approach.

This study confirms the increased risk of a difficult split if lower third molars are present, however no single anatomical difficulty. A simple classification of the typical patterns of unfavourable splits that of difficult sagittal split osteotomies and measurement contributed to the level of may occur when a sagittal split osteotomy is performed is proposed.

\section{Funding}

None.

\section{Competing interest}

None.

\section{Ethical approval}

Ethical approval was obtained from the Human Research Ethics Committee (Medical). Clearance certificate: M0050341.

\section{References}

1. Blair VP. Operations on the jaw bone and face. Surg Gynecol Obstet 1907;4:67-8.

2. Trauner R, Obwegeser H. The surgical correction of mandibular prognathism and retrognathia with consideration of genioplasty. Oral Surg Oral Med Oral Pathol 1957; 10:677-89.

3. Dal Pont G. Retromolar osteotomy for the correction of prognathism. J Oral Surg 1961; 19:42-7.

4. Hunsuck EE. A modified intraoral sagittal splitting technique for correction of mandibular prognathism. J Oral Surg Anaesth 1968; 2:249-52.

5. Epker BN. Modifications in the sagittal osteotomy of the mandible. J Oral Surg 1977;35:157-9.

6. Macintosh RB. Experience with the sagittal split osteotomy of the mandibular ramus: a 13-year review. J Maxillofac Surg 1981;9: 151-65.

7. Behrman SJ. Complications of the sagittal osteotomy of the mandibular ramus. J Oral Surg 1972;30:554-61. 
8. Guernsey LH, De Champlain RW. Sequelae and complications of the intra-oral sagittal osteotomy in the mandibular ramus. Oral Surg Oral Med Oral Pathol 1971;32:176-92.

9. Precious DS, Lung KE, Pynn BR, Goodday $\mathrm{RH}$. Presence of impacted teeth as a determining factor of unfavorable splits in 1256 sagittal osteotomies. Oral Surg Oral Med Oral Pathol 1998;85:362-5.

10. Reyneke JP, Ferretti C. Intra-operative diagnosis of condylar sag after bilateral sagittal split ramus osteotomy. Br J Oral Maxillofac Surg 2002;40:285-92.

11. Turvey TA. Intraoperative complications of sagittal split osteotomy of the mandibular ramus: Incidence and management. $J$ Oral Maxillofac Surg 1985;43:504-9.

12. Witherow H, Offord D, Eliahoo J, Stewart A. Postoperative fractures of the lingual plate after bilateral sagittal split osteotomies. $\mathrm{Br} \mathrm{J}$ Oral Maxillofac Surg 2006;44:296-300.

13. Reyneke JP, Tsakiris P, Becker P. Age as a factor in the complication rate after removal of unerupted/impacted third molars at the time of mandibular sagittal split osteotomy. J Oral Maxillofac Surg 2002;60:654-9.

14. Mehra P, Castro V, Freitas RZ, Wolford LM. Complications of the mandibular sagittal split osteotomy associated with the presence or absence of third molars. J Oral Maxillofac Surg 2001;59:854-8. 